NATIONALSOZIALISTISCHE GROSSRAUMORDNUNG 


\section{SCHR IFTENREIHE}

\section{DER VIERTELJAHRSHEFTE FÜR ZEITGESCHICHTE}

NUMMER 4

Im Auftrag des Instituts für Zeitgeschichte

herausgegeben von Hans Rothfels und Theodor Eschenburg

Redaktion: Martin Broszat 


\section{NATIONALSOZIALISTISCHE GROSSRAUMORDNUNG}

Die Konstruktion einer »deutschen Monroe-Doktrin « 
(c) 1962 Deutsche Verlags-Anstalt GmbH., Stuttgart. Gesetzt aus der Monotype Walbaum-Antiqua. Gesamtherstellung: Deutsche VerlagsAnstalt GmbH., Stuttgart. Printed in Germany 


\section{IN HALT}

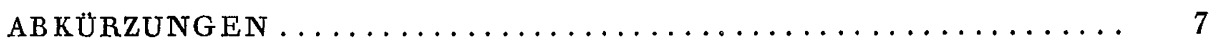

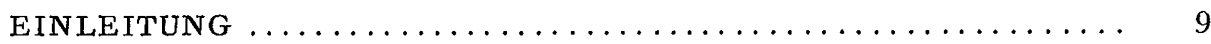

I DIE KONSTRUKTION EINER „DEUTSCHEN MONROE-DOKTRIN“

1. Der außenpolitische Fintergrund $\ldots \ldots \ldots \ldots \ldots \ldots \ldots \ldots \ldots \ldots \ldots \ldots$

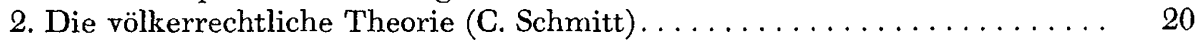

II GROSSRAUMPOLITIK GEGEN INTERVENTION VON AUSSEN

1. Die ursprüngliche Monroe Doctrine und die Heilige Allianz . . . . . . . 28

2. Nationalsozialistische Großraumpolitik und die angelsächsischen Mächte... 32

3. Theorie der Großraumordnung - Antithese zum „universalen Inter-

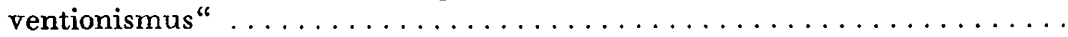

III DIE STAATENBEZIEHUNGEN INNERHALB DES GROSSRAUMES

1. Monroe Doctrine und amerikanische Staatenwelt. . . . . . . . . . 66

2. Die nationalsozialistische "Neue Ordnung Europas" $\ldots \ldots \ldots \ldots \ldots \ldots \ldots 71$

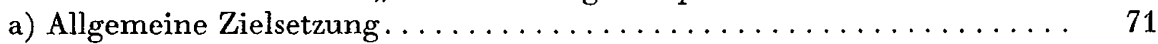

b) Die „Neuordnung “ für die besetzten west- und nordeuropäischen Gebiete (Frankreich, Luxemburg, Belgien, die Niederlande, Dänemark,

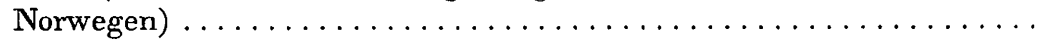

c) Unterwerfung und bevölkerungspolitische Ziele in den besetzten Ostgebieten (Polen, „Protektorat Böhmen und Mähren“, baltische Staaten,

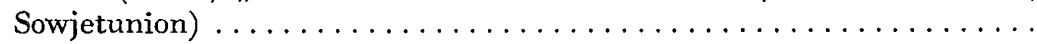

d) Finnland und die abhängigen Staaten im Südosten (Slowakei, Ungarn, Rumänien, Bulgarien, Jugoslawien, Griechenland) . . . . . . . . . 103

e) Der bevormundete italienische Bundesgenosse . . . . . . . . . 109

f) Die Neutralen (Schweden, Schweiz, Spanien, Portugal) . . . . . . . . 111

g) Ideologische Ausrichtung in Hitlers Großraum (Judenpolitik) . . . . . . 113

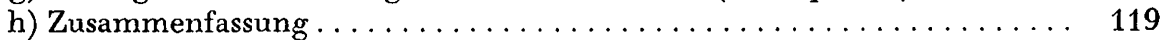

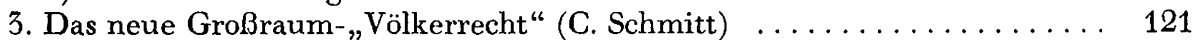

IV DIE NATIONALSOZTALISTISCHE FEHLDEUTUNG DER MONROE DOCTRINE - MISSVERSTÄNDNIS DER GRUNDLAGEN AMERTKANISCHER AUSSENPOLITIK

1. Falsche völkerrechtswissenschaftliche Interpretation . . . . . . . . . 146

2. Kontinentale Mißdeutung der geopolitischen Aspekte der US-Außenpolitik 149

3. Der Isolationismus und der amerikanische Imperialismus der Jahrhundertwende als Vorbilder der nationalsozialistischen Großraumdoktrin . . . . . . . . 


\section{ABKÜRZUNGEN}

AA

ADAP

AdG

AGRA

AJIL

AöR

DAFR

DBFP

DDP

DEVLAG

DIA

DNSAP

Doc./Dok.

DR

DRWs

IfZ

IMT/IMG

Nbg./Nürnbg. Dok. Nürnberger Dokument (gelegentlich auch nur mit den Buchstaben der Serien : C, D, EC, F, L, NG, NO, PS, R, RF, UK, USSR bezeichnet)

NS

NSB

Auswärtiges Amt

Akten zur Deutschen Auswärtigen Politik 1918-1945. Aus dem Archiv des Auswärtigen Amtes. Serie D (1937-1945) BadenBaden 1950-1951

Archiv der Gegenwart. Herausgeber, Eigentümer und Verleger : Archiv der Gegenwart Gesellschaft mbH. Wien

Amis du Grand Reich Allemand

The American Journal of International Law. A quarterly. With suppl. section of documents. Publ. by the American Society of International Law. New York-Washington

Archiv des öffentlichen Rechts. Herausgegeben von W. Grewe, E. Jacobi, W. Jellinek, E. Kaufmann, H. Loening, K. Schmid, R. Smend, E. Walz, Tübingen

Documents on American Foreign Relations, edited by S. Shepard Jones and Denys P. Myers, World Peace Foundation, Boston 1939-1942

Documents on British Foreign Policy 1919-1939, Third Series, edited by E. L. Woodward and Rohan Butler. Vol. IV. London 1951

Dokumente der Deutschen Politik. Reihe: Das Reich Adolf Hitlers, herausgegeben von F. A. Six, Berlin 1942-1944

Deutsch-Vlämische Arbeitsgemeinschaft

Documents on International Affairs, edited by John W. WheelerBennett, Oxford-London 1929-1935; edited by the Royal Institute of International Affairs London-New-York-Toronto 1951-1954

Dansk National Socialistiske Arbejder Parti

Dokument

Deutsches Recht (Ausgabe A). Zeitschrift des Bundes Nationalsozialistischer Deutscher Juristen, vereinigt mit Juristische Wochenschrift. Herausgegeben von Hans Frank. Zentralorgan des NS-Rechtswahrerbundes. Berlin-Leipzig-Wien

Deutsche Rechtswissenschaft. Vierteljahresschrift der Akademie für Deutsches Recht. Herausgegeben von der Abt. für Rechtsforschung der Akademie für Deutsches Recht. Hamburg.

Institut für Zeitgeschichte

Der Prozeß gegen die Hauptkriegsverbrecher vor dem Internationalen Militärgerichtshof, Nürnberg, 14. November 1945 bis 1. Oktober 1946, Nürnberg 1947-1949

nationalsozialistisch; auch: Nasjonal Samling

Nationaal Socialistische Beweging 
NSNAP

OAS

Ob.d.H.

OKW

RAM

RVL

SSHA

VERDINASO

VfZ

VNV

ZAkDR

ZаöRVR/ZRVR

ZföR

Zft.f.d.ges.StWs

$\mathrm{ZfV/ZfVR}$
Nationaal Socialistische Nederlandsche Arbeiderspartij

Organization of American States

Oberbefehlshaber des Heeres

Oberkommando der Wehrmacht

Reichsaußenminister

Reich-Volksordnung-Lebensraum. Zeitschrift für völkische Verfassung und Verwaltung. Herausgegeben von W. Stuckart, W. Best, G. Klopfer, R. Lehmann, R. Höhn. Darmstadt

SS-Hauptamt

Verbond van Dietsche Nationaal Solidaristen

Vierteljahrshefte für Zeitgeschichte. Im Auftrag des Instituts für Zeitgeschichte München herausgegeben von Hans Rothfels und Theodor Eschenburg, Stuttgart

Vlaamsch National Verbond

Zeitschrift der Akademie für Deutsches Recht. Herausgegeben von H. Frank. München und Berlin

Zeitschrift für ausländisches öffentliches Recht und Völkerrecht. Herausgegeben vom Institut für ausländisches öffentliches Recht und Völkerrecht Berlin. Berlin

Zeitschrift für öffentliches Recht. Herausgegeben von E. Bernatzik, H. Kelsen. Berlin-Leipzig-Wien

Zeitschrift für die gesamte Staatswissenschaft. Herausgegeben von F. Böhm, W. Grewe u. a. Tübingen

Zeitschrift für Völkerrecht. Begründet von J. Kohler, herausgegeben von M. Fleischmann, K. Strupp. Trebnitz 


\section{EINLEITUNG}

Das Bestreben, die Prinzipien der amerikanischen Monroe Doctrine auf andere Erdteile zu übertragen, ist nicht neu. Ein Beispiel ist die auch in der Literatur oft erörterte sogenannte „japanische Monroe-Doktrin“. Die hier vorgelegte Untersuchung setzt sich mit ihrem europäischen Analogon auseinander: mit dem Versuch, die nationalsozialistische Großraumpolitik in Europa unter Hinweis auf das Vorbild der Monroe Doctrine zu rechtfertigen und aus dieser Doktrin die Aufteilung der Erde in sogenannte Großräume unter jeweiligen Führungsmächten abzuleiten. Die Berufung auf die Monroe Doctrine mit dem sehr durchsichtigen Ziel, die USA aus dem von Hitler begonnenen Eroberungskrieg in Europa herauszuhalten, war dabei nur eine der verschiedenen Spielarten nationalsozialistischer Großraumtheorien. Teilweise stellten diese eine Weiterbildung und Modifizierung, vor allem auch räumliche Erweiterung des Mitteleuropa-Gedankens dar, wie ihn Friedrich Naumann, Ernst Jäckh u. a. konzipiert hatten. Insbesondere von der geopolitischen Schule war die Theorie eines "Großlebensraums" entwickelt worden, der Gedanke einer autarkistischen wirtschaftspolitischen Zusammenfassung mehrerer Völker unter deutscher Führung und ihrer politisch-strategischen Abgrenzung nach außen (G. Jentsch u. a.). Auch dabei wurde auf das geopolitische Modell der Monroe Doctrine zurückgegriffen, während andererseits völkische Theoretiker (W. Daitz, R. Höhn) die überwiegend geographisch und politisch bestimmte amerikanische Doktrin nicht als Vorbild gelten ließen und stattdessen von „biologischen MonroeDoktrinen " ausgingen, d. h. in der biologischen Substanz eines Großraumes den bestimmenden Faktor für seine Ausdehnung und Gestalt sahen. Nicht diese völkischen und geopolitischen Konzeptionen im engeren Sinne sind aber Gegenstand unserer Untersuchung, sondern jene Thesen, welche die angestrebte Hegemonie und Expansion des Dritten Reiches zu "verrechtlichen " suchten, d. h. die "Großraumordnung" als neues Völkerrechtsprinzip ausgaben. An erster Stelle steht dabei die völkerrechtswissenschaftliche Theorie, die der Staats- und Völkerrechtler Carl Schmitt entwarf, als die nationalsozialistische Außenpolitik in ihre imperialistische Phase eintrat. Carl Schmitt sah im Kerngedanken der ursprünglichen Monroe Doctrine, dem Interventionsverbot für „raumfremde Mächte“, ein Grundprinzip für ein neues Völkerrecht, den "Präzedenzfall" einer völkerrechtlichen Großraumordnung, der auf andere Räume und Situationen übertragbar sei. Er entwickelte daraus die These vom Großraum, der seine innere Ordnung von einer „raumzugehörigen" führenden Macht erhält, dem „Reich" als Ordnungsmacht und Träger der neuen Völkerrechtsordnung.

Unsere Untersuchung hat zu erweisen, inwieweit die nationalsozialistische Außenpolitik und Völkerrechtstheorie, sofern sie sich auf die Monroe Doctrine 
berief, deren Grundsätze bewußt oder unbewußt fehldeutete und verfälschte. Eine vergleichende Betrachtung der amerikanischen Doktrin und ihres nationalsozialistischen Gegenstückes muß an zwei Zentralpunkten ansetzen: bei der Untersuchung des Interventionsbegriffes für die "raumfremden " und des Zuordnungsverhältnisses der "raumzugehörigen“ Staaten. Dieser Vergleich aber kann nicht nur im Abwägen der beiderseitigen Doktrinen bestehen, er wird ebenso die Praxis der nationalsozialistischen Großraumpolitik und ihre erkennbaren Intentionen an dem angeblichen amerikanischen Vorbild zu messen haben. Es wird dabei die „ideale“ Zweckbestimmtheit des von Carl Schmitt entworfenen „neuen Völkerrechts" sichtbar werden: die Umdeutung des Interventionsverbots zu einem Mittel der Abschirmung deutscher Aggression und Gewaltherrschaft, die sinnverkehrende Anwendung des Reichsbegriffs, durch welche die faktische Machtüberlegenheit zum Rechtsinstitut erhoben werden sollte. Aus dieser Analyse wird sich aber auch ergeben, daß die nationalsozialistische Außenpolitik mit ihrer, kontinentalem Denken entspringenden, Mißdeutung der amerikanischen Monroe Doctrine unwillentlich selbst gerade die Voraussetzungen für eine amerikanische Nichteinmischungspolitik zerstörte und damit ihr eigenes Ziel - die Abgrenzung des Geltungsbereichs einer „deutschen " und der amerikanischen Monroe-Doktrin, d. h. die Fernhaltung der USA aus dem europäischen Machtraum des Dritten Reiches - zum Scheitern brachte.

Die vorliegende Abhandlung ist aus einer Dissertation an der Philosophischen Fakultät der Freien Universität Berlin hervorgegangen. Die mit dem Rahmen dieser Schriftenreihe gegebene Umfangbegrenzung machte es nötig, den ursprünglichen Text verschiedentlich zu kürzen und auf einige spezielle Gesichtspunkte, insbesondere auch die Erörterung der völkischen und geopolitischen Großraumtheorien zu verzichten. Der besondere Dank des Verfassers gilt seinem langjährigen Lehrer, Herrn Prof. Ernst Fraenkel, der diese Untersuchung anregte, und Herrn Dr. Martin Broszat, der bei ihrer Kürzung und Umarbeitung für die vorliegende Veröffentlichung wertvolle Ratschläge gab. 
Die Berufung auf das amerikanische Vorbild der Monroe-Doktrin zur Abschirmung nationalsozialistischer Aggressionspolitik und Gewaltherrschaft in Europa spielte in der Diplomatie des Dritten Reiches und in der gleichzeitigen Theorie zur Rechtfertigurg eines deutschen Großraumes eine zentrale Rolle. Durch einen exakten analytischen Vergleich arbeitet Gruchmann die fundamentalen Unterschiede zwischen den Grundlagen der Monroe-Doktrin und der nationalsozialistischen Großraumpolitik heraus. Der Autor gibt einen prägnanten Überblick über die Politik der sogenannten nationalsozialistischen Neuordnung in Europa und die Stellung, die den einzelnen im Zweiten Weltkrieg unterworfenen Ländern und den verbündeten Satellitenstaaten darin zugedacht war. Er verbindet damit eine eingehende Auseinandersetzung mit der ,,völkerrechtlichen Großraumordnung" des Staats- und Völkerrechtlers Carl Schmitt, die diese Hegemonialpolitik opportunistisch zu rechtfertigen suchte.

Die nationalsozialistische Fehldeutung der Monroe-Doktrin macht das grundsätzliche Mißverständnis amerikanischer Außenpolitik sichtbar. Ein wesentlicher Grund dafür, daß Hitler schließlich unwillentlich selbst das Eingreifen der USA in Europa provozierte, das er unter Berufung auf die Monroe-Doktrin zu verhindern suchte.

Autor: Dr. Lothar Gruchmann, geb. 25.1.1929 in Plauen im Vogtland, studierte in Berlin Politikwissenschaft, neuere Geschichte und Staatsrecht und ist seit 1960 wissenschaftlicher Mitarbeiter am Institut für Zeitgeschichte in München.

Veröffentlichungen: „Das Korea-Problem - Seine Bedeutung für das geteilte Deutschland", „Völkerrecht und Moral. Ein Beitrag zur amerikanischen Neutralitätspolitik 1939-1941“. 
Schriftenreihe der Vierteljahrshefte für Zeitgeschichte

IN VORBEREITUNG SIND:

CONRAD F. LATOUR

Südtirol und die Achse Berlin - Rom

1938-1945

\section{Militäropposition \\ 1938 - 1940}

Tagebücher von Oberstleutnant Helmuth Groscurth, herausgegeben von Harold Deutsch und Helmut Krausnick

ENNO GEORG

\section{Die wirtschaftlichen}

Unternehmungen der SS

RUDOLF HEBERLE

Die politische Willensbildung in Schleswig-Holstein vor 1933

Jährlich erscheinen zwei Veröffentlichungen

DEUTSCHE VERLAGS-ANSTALT STUTTGART 